\title{
SPECTRAL CHARACTERISTICS OF THE SUPRAMOLECULAR COMPLEXES OF POLYPYRROLIC SENSITIZERS AND CYCLODEXTRIN CARRIERS: USAGE IN PHOTODYNAMIC THERAPY OF TUMORS
}

\author{
Martin Huf ${ }^{1}$, Hana Kolářová ${ }^{1}$, Robert Bajgar ${ }^{1}$, Jaroslav Maceček ${ }^{1}$, Marek Tomečka ${ }^{1}$, Pavla Nevřelová1 ${ }^{\text {, Jiři Mosinger }}$, \\ Pavel Tomek ${ }^{3}$, Miroslav Strnad ${ }^{4}$
}

Palacky University Olomouc, Faculty of Medicine, Czech Republic: Institute of Medical Biophysics ${ }^{1}$; Charles University in Prague, Faculty of Science, Czech Republic: Insitute of Inorganic Chemistry²; Palacky University Olomouc, Faculty of Science, Czech Republic: Institute of Experimental Physics ${ }^{3}$, Laboratory of Growth Regulators ${ }^{4}$

Summary: The objective of our work was to describe the photophysical properties (absorption and fluorescence) of the sensitizers TPPS , ZnTPPS $_{4}$ a PdTPPS 4 and above all the complexes of these sensitizers with cyclodextrin carriers HP$\alpha$-CD, HP- $\beta$-CD and HP- $\gamma$-CD (2-hydroxypropyl- $\alpha, \beta, \gamma$-cyclodextrin) in a suitable environment for the cultivation of cancerous cell lines, and to determine the optimal radioactive conditions for maximizing photodynamic effects in cancerous cells.

Key words: Polypyrrolic sensitizer; Cyclodextrin carrier; PDT; Absorption spectrum; Fluorescence spectrum

\section{Introduction}

Photodynamic therapy (PDT) has lately been involved in the treatment of oncological, cardiovascular, skin and eye diseases. PDT is founded on the use of sensitizers, which have a longer retention time in cancerous cells. During the photoactivity of sensitizers, using visible radiation of suitable wavelength, the generation of cytoxic material is occured which leads to irreversible damage in cancerous cells. In contrast to conventional treatment methods (surgery, radiotherapy and chemotherapy) PDT allows selective removal of cancerous cells without damaging the surrounding healthy tissue $(1,2,7,8)$.

Today the quite often used sensitizer is Photofrin II. This sensitizer is one of the so-called „first generation sentiziers", which sets the standard for its class. Above all it's selectivivity is too narrow. Furthermore, thanks to it's narrow extinction coefficient it is necessary to use a large amount of the sensitizer to achieve a photodynamic reaction.

The above mentioned problems lead to the creation socalled „second generation sensitizers“. This includes porfyrin, phtalocyanines, naphtalocyanines, chlorins, and also polypyrrolic sensitizers TPPS,$Z_{4}$ TPPS 4 a PdTPPS „Second generation sensitizers" are considerably more selective which enhances the effectivity of PDT $(3,5,6)$. Naturally they are hydrophobic, so they disolve poorly in water. This problem can be solved by the use of a suitable sensitizer carrier (polymer particles, liposomes, antibodies, etc.).

Cyclodextrins (CD) are currently under intensive study as one of the suitable carriers for this entire class of medicine, and also of photodynamically active materials. Cyclodextrins are cyclic oligosacharades, compounded of 6,7 a 8 $(\alpha, \beta \alpha \gamma)$ a - 1, 4-D - glucopyranose units. Photodynamically active materials are bound in the cyclodextrin cavity with the help of hydrophobic and van der Waals forces. This binding with the CD allows a hydrophobic sensitizer to be transported in a water based medium. CD also has a significant monomerization effect, which inhibits the aggregation of sensitizers and increases the quantum yield and life span of the excited state of the sensitizer (4).

\section{Material and methods}

The absorption spectra of the cultivation medium (DMEM), the selected solution of porfyrin sensitizers TPPS4, ZnTPPS4 and PdTPPS 4 and these sensitizers with cyclodextrin carriers HP- $\alpha-C D$, HP- $\beta-C D$ and HP- $\gamma-C D$ were measured with spectrophotometer UNICAM UV 550. Flourescent spectra were obtained with the use of spectrofluorometer HITACHI F4500. The cultivation medium was used as the solvent. The concentration of the sensitizer was $10 \mu \mathrm{M}$, cyclodextrin carrier was $1 \mathrm{mM}$, thus cyclodextrin was compared with the sensitizers in hundredfold concentration excess. 


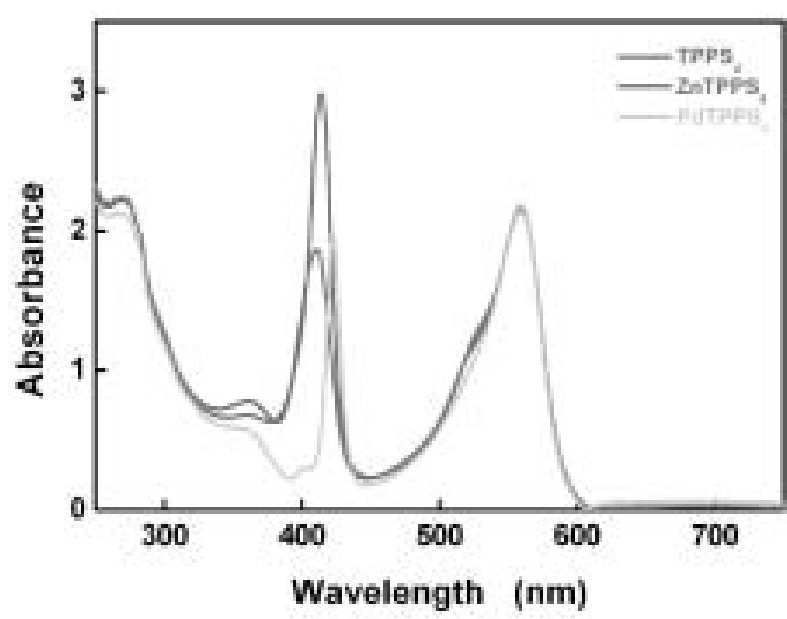

Fig. 1: Absorption spectra of $10 \mu \mathrm{M}$ sensitizers.

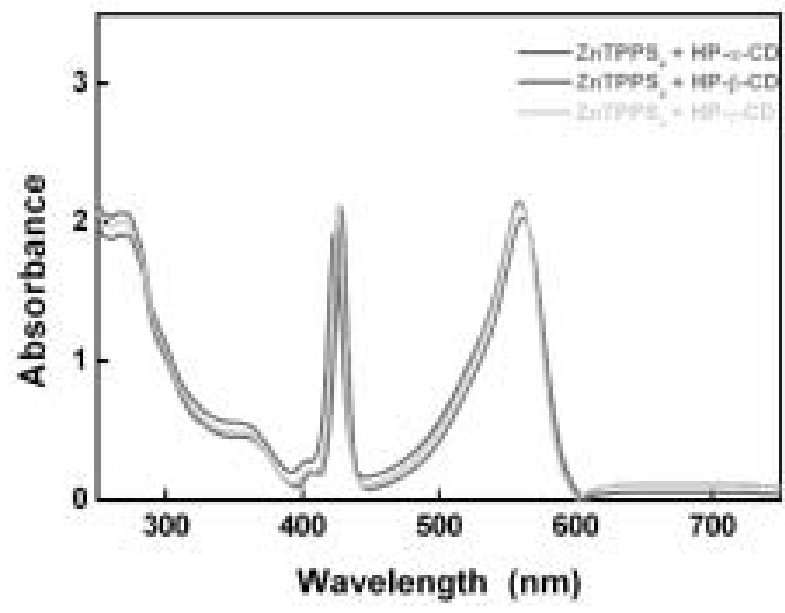

Fig. 2: Absorption spectra of $10 \mu \mathrm{M} \mathrm{ZnTPPS}_{4}$ bound to 1 $\mathrm{mM}$ cyclodextrin carriers.

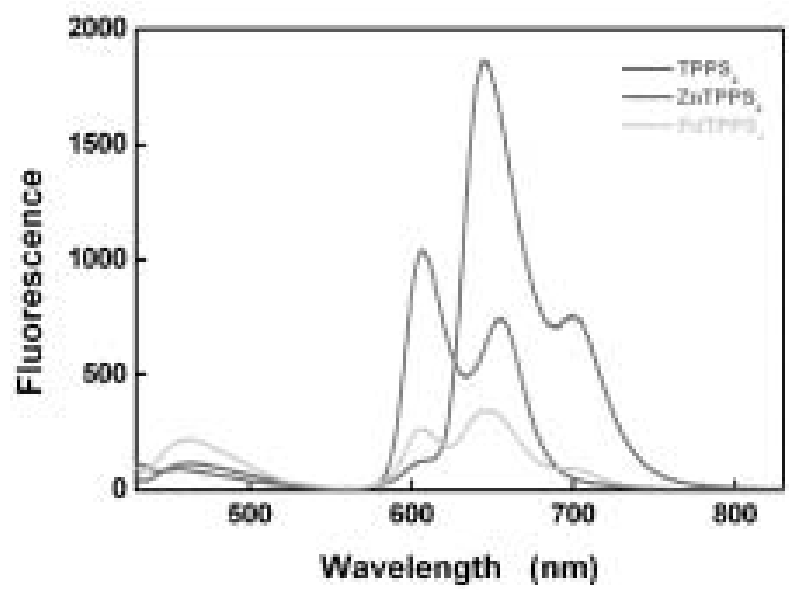

Fig. 3: Fluorescence emission spectra of $10 \mu \mathrm{M}$ sensitizers. Exciting wavelengths are the same like wavelengths of absorption maxima of sensitizers in the Soret region.

\section{Results}

Fig. 1 shows the absorption spectrum of the polypyrrolic sensitizer solution. The wavelength absorption maximum for sensitizer TPPS 4 is $415 \mathrm{~nm}$. The sensitizer $\mathrm{ZnTPPS}_{4}$ expresses a shift in absorption maximum to the short wave length of $2 \mathrm{~nm}$, thus the level of $413 \mathrm{~nm}$. The absorption maximum of sensitizer PdTPPS $_{4}$ is near the wavelength 423 nm. Fig. 2 shows the absorption spectra of the sensitizer ZnTPPS4 in combination with the cyclodextrin carriers, which demonstrates that the type of used carrier only slightly affects the form of the spectrum.

In Fig. 3 the fluorescent emission spectra are obtained near the wavelength which corresponds to the sensitizer absorption maximum in the Soret region. The spectra illustrate a strong difference in the location of the maxima and the fluorescence intensity, where as with all of sensitizers there emerges two distinct fluorescent bands. TPPS 4 shows the highest fluorescence. The fluorescence intensity is near that of other sensitizers, after which sensitizers ZnTPPS $_{4}$ a PdTPPS $_{4}$ diminish along with it, such that it highlights the longer wavelength maximum with regard to the maximum short wavelength. Sensitizer PdTPPS $_{4}$ gains a long wavelength band with a higher intensity of fluorescence. Individual sensitizers also differ in the position of their flourescent maxima. Fluorescent emission spectra of the sensitizer ZnTPPS4 in combination with the cyclodextrin carriers, which demonstrates that the type of used carrier only slightly affects the form of the spectrum. We found similar behavior in the recordings of the spectra for sensitizers TPPS $_{4}$ a PdTPPS $_{4}$.

\section{Discussion}

The absorption spectra were measured in conditions parallel to that of the cultivated tumor cells. The spectra were measured in the cultivation medium; its character can influence the resulting exposure parameters because a portion of active radiation will likely be asborbed by the cultivation medium itself. The absorption spectra of the sensitizer solutions differ in absorption spectra of the cultivation media only in the region of short wavelength of light. Minor differences in the spectra of different sensitizers in this area fit their individual chemical structure (Fig. 1). The absorption of the sensitizer solutions in the ultraviolet area of the electromagnetic spectrum and the area around the wavelength 560 $\mathrm{nm}$ pertains to its cultivation medium, which was used as the solvent. The cyclodextrin carrier caused a shift in the absorption maxima of individual sensitizer solutions (Fig. 1 and 2). If we take a sensitizer in a cyclodextrin carrier then the absorption spectra of these complexes will not differ from the type of carrier used (Fig. 2). The fluorescent spectra of the sentiziters differ in their fluorescent intensity, in the long wavelength maxima (Fig. 3). These differences are attributed to their individual chemical structure. 


\section{Conclusion}

The study of photophysical properties allows the establishment of irradiation parameters and conditions of the cultivation of cancer cells for combined studies of the cytotoxicity and phototoxicity of sensitizers bound in cyclodextrin carriers in vitro methods.

\section{Special thanks}

This work was supported by a grant from the Palacky University (grant no. 11101101), the GAČR grant no. 203/ 02/1483, and research plan MŠMT no. 153100008.

\section{References}

1. Jori G. In vivo transport and pharmacokinetic behavior of tumour photosensitizers. Ciba Found Symp 1989;146:78-86.

2. Konan YN, Gurny R, Allemann E. State of the art in the delivery of photosensitizers for photodynamic therapy. J Photochem Photobiol B. 2002;66(2): 89-106.
3. Leach MW, Higgins RJ, Autry SA, Boggan JE, Lee SJ, Smith KM. In vitro photodynamic effects of lysyl chlorin p6: cell survival, localization and ultrastructura changes. Photochem Photobiol 1993;58(5):653-60.

4. Moan J. Properties for optimal PDT sensitizers. J Photochem Photobiol B. 1990;5(3-4):521-4.

5. Mosinger J, Kliment V Jr, Sejbal J, Kubat P, Lang K. Host - guest complexes of anionic porphyrin sensitizers with cyclodextrins. J Porphyrins Phthalocyanines 2002;6:513-23.

6. Ochsner M. Photodynamic therapy: the clinical perspective. Review on applications for control of diverse tumorous and non-tumorous diseases. Arzneimittelforschung 1997;47(11):1185-94.

7. Strauss WS, Gschwend $M H$, Sailer $R$, Schneckenburger $H$, Steiner $R$, Ruck A. Intracellular fluorescence behaviour of meso - tetra (4-sulphonatophenyl) porphyrin during photodynamic treatment at various growth phases of cultured cells. J Photochem Photobiol B. 1995;28:155-61.

8. Wood SR, Holroyd JA, Brown SB. The subcellular localization of $\mathrm{Zn}$ (II) phthalocyanines and their redistribution on exposure to light. Photochem Photobiol 1997;65:397-402.

Mgr. Martin Huf, Palacky University Olomouc, Faculty of Medicine, Institute of Medical Biophysics, Hněvotínská 3, 77515 Olomouc, Czech Republic. e-mail: m.huf@seznam.cz 